\title{
SOME RELATIONS BETWEEN VARIATIONAL-LIKE INEQUALITIES AND EFFICIENT SOLUTIONS OF CERTAIN NONSMOOTH OPTIMIZATION PROBLEMS
}

\author{
LUCELINA BATISTA SANTOS, MARKO A. ROJAS-MEDAR, \\ AND ANTONIO RUFIÁN-LIZANA
}

Received 28 November 2005; Revised 4 June 2006; Accepted 21 June 2006

We study the equivalence between the solutions of the variational-like inequality problem and the solutions of certain nonsmooth and nonconvex vectorial optimization problem.

Copyright (c) 2006 Hindawi Publishing Corporation. All rights reserved.

\section{Introduction}

The connections between variational inequalities and optimization problems is well known, and many investigators have discussed them along many years; see, for instance, $[1,8,10,13]$. This last article, which was authored by Giannessi, in particular, is one of the main works that study these connections in the finite-dimensional context. In recent years, the interest in the investigation on the relationships between these two classes of problems has increased, resulting in several different conditions for the existence of solutions for many variational-type inequalities (e.g., $[5,16,19,20])$. Connections among variational inequalities and vectorial optimization problems have also been studied in $[11,18,25]$, for instance.

By using a variational-like inequality, Lee et al. [19] obtained some results of existence of solutions for nonsmooth invex problems, which are generalizations of those obtained by Chen and Craven [4] for differentiable convex problems. Recently, Giannessi [11] showed the equivalence between efficient solutions of a differentiable and convex optimization problem and the solutions of a variational inequality of Minty type. He also proved the equivalence between weak efficient solutions of a differentiable convex optimization problem and solutions of a variational inequality of weak Minty type.

Following this last line of investigation, Lee [17] was able to establish the equivalence between the solutions of the inequalities of Minty and Stampacchia types for subdifferential (in the convex analysis sense) and efficient solutions and weakly efficient solutions, respectively, in the case of vectorial nonsmooth convex optimization problems. Moreover, using these characterizations, he proved a theorem on existence of weakly efficient solutions for the vectorial nonsmooth convex optimization problem, under hypothesis of compactness. 
In this work, we extend the results obtained early by Lee [17] for the nonsmooth invex context.

This paper is organized as follows. In Section 2, we fix some basic notation and terminology. In Section 3, we prove some connections between efficient solutions and vectorial optimization problems; in Section 4, we consider the case of weakly efficient solutions. Finally, in Section 5, we use the results of the previous sections to show an existence result of the weakly efficient solutions of nonsmooth invex vectorial optimization problem, under weak hypothesis of compactness.

\section{Preliminaries}

In this section we recall some notions of nonsmooth analysis; for more details, see, for instance, Clarke [6].

Let $\mathbb{R}^{n}$ be the $n$-dimensional Euclidean space and $\mathbb{R}_{+}^{n}$ its nonnegative orthant. In the sequel $\Omega$ will be a nonempty open subset of $\mathbb{R}^{n}$. A function $f: \Omega \rightarrow \mathbb{R}$ is said to be Lipschitz near $x \in \Omega$ if, for some $K>0$,

$$
|f(y)-f(z)| \leq K\|y-z\|
$$

for all $y, z$ within some neighborhood of $x$. We say that $f$ is locally Lipschitz on $\Omega$ if $f$ is Lipschitz near any given point of $\Omega$. The generalized directional derivative of $f$ at $x$ in the direction $v$, denoted by $f^{0}(x, v)$, is defined as follows:

$$
f^{0}(x, v)=\lim \sup _{\substack{y \rightarrow x \\ t \downarrow 0}} \frac{1}{t}[f(y+t v)-f(y)] .
$$

The generalized gradient of $f$ at $x$, denoted by $\partial f(x)$, is the subset of $\mathbb{R}^{n}$ given by

$$
\partial f(x)=\left\{\xi \in \mathbb{R}^{n}: f^{0}(x, v) \geq\langle\xi, v\rangle, \forall v \in \mathbb{R}^{n}\right\}
$$

where $\langle\cdot, \cdot\rangle$ is the usual scalar product in $\mathbb{R}^{n}$. The set $\partial f(x)$ is nonempty when $f$ is Lipschitz near $x \in \Omega$.

Let $X$ be a nonempty subset of $\mathbb{R}^{n}$. The distance function related to $X$ is the function $d_{X}: \mathbb{R}^{n} \rightarrow \mathbb{R}$, defined by

$$
d_{X}(x)=\inf \{\|x-y\|: y \in X\}
$$

The distance function is not differentiable everywhere but is globally Lipschitz.

Let $x \in X$; a vector $v \in \mathbb{R}^{n}$ is said to be tangent to $X$ at $x$ if $d_{X}^{0}(x, v)=0$ and the set of the tangent vectors to $X$ at $x$ is called Clarke tangent cone and denoted by $T_{X}(x)$. This set is a closed convex cone in $\mathbb{R}^{n}$. The Clarke normal cone to $X$ at $x$ can be defined by polarity with $T_{X}(x)$ :

$$
N_{X}(x)=\left\{\xi \in \mathbb{R}^{n}:\langle\xi, v\rangle \leq 0, \forall v \in T_{X}(x)\right\}
$$


Suppose that $f$ is a locally Lipschitz function on $\Omega$ and attains a minimum over $X$ at $\bar{x}$. Then,

$$
0 \in \partial f(\bar{x})+N_{X}(\bar{x})
$$

We say that $\bar{x} \in X$ is a Clarke stationary point of $f$ over $X$ if (2.6) holds.

Hanson [14] considered the differentiable functions $f: \mathbb{R}^{n} \rightarrow \mathbb{R}$ such that for all $x, y \in$ $\mathbb{R}^{n}$, there exists $\eta(y, x) \in \mathbb{R}^{n}$ such that

$$
f(y)-f(x) \geq\langle\nabla f(x), \eta(y, x)\rangle .
$$

Nowadays, such functions are generally known as invex functions due to Craven and Glover [7], who first named them so. This invexity notion generalizes the concept of convexity and allows to extend sufficient conditions of optimality and duality results to nonconvex optimization problems (see, e.g., $[2,12,21]$ ). Invexity has now been extended to nondifferentiable locally Lipschitz functions. See, for example, Craven and Glover [7], Reiland [23], and Phuong et al. [22]. We use the definition provided in [22]: let $X$ be a nonempty subset of $\Omega$ and suppose that $f: \Omega \rightarrow \mathbb{R}$ is a locally Lipschitz function on $\Omega$. We say that $f$ is invex on $X$ if, for every $x, y \in X$, there is $\eta(y, x) \in T_{X}(x)$ such that

$$
f(y)-f(x) \geq f^{0}(x, \eta(y, x)) .
$$

The above notion of invexity is very powerful because it allows to treat smooth and nonsmooth constrained problems, in the presence of an abstract constraint. In later sections we will deal with this subject.

An important result obtained by Phuong et al. is the following invexity characterization; see [22].

Proposition 2.1. Let $X$ be a nonempty subset of $\Omega$. A locally Lipchitz function $f$ is invex on $X$ if and only if every Clarke stationary point of $f$ over $X$ is a global minimum.

\section{Efficient solutions and variational-like inequalities}

Let $X$ be a nonempty subset of $\mathbb{R}^{n}$ and let $f_{i}: \mathbb{R}^{n} \rightarrow \mathbb{R}, i=1, \ldots, p$, be the given functions. We consider the following vectorial optimization problem:

$$
\text { minimize } f(x):=\left(f_{1}(x), \ldots, f_{p}(x)\right) \quad \text { subject to } x \in X \text {. }
$$

As it is well known, differently from the case of scalar optimization problems, there is not a unique concept of solution for vectorial optimization problems. Amongst the numerous definitions of solutions for such problems existing in the literature, we will consider the followings.

Definition 3.1. A point $y \in X$ is said to be

(i) an efficient solution of (P) if there is no other point $x \in X$ such that $f(x) \leq f(y)$, or equivalently,

$$
f(x)-f(y) \notin-\mathbb{R}_{+}^{n} \backslash\{0\}, \quad \forall x \in X
$$


(ii) a weakly efficient solution of (P) if there is no other point $x \in X$ such that $f(x)<$ $f(y)$, or equivalently,

$$
f(x)-f(y) \notin-\operatorname{int} \mathbb{R}_{+}^{n}, \quad \forall x \in X ;
$$

(iii) a properly efficient solution of (P) if it is efficient and if there exists $M>0$ such that for each $i$,

$$
\frac{f_{i}(x)-f_{i}(y)}{f_{j}(y)-f_{j}(x)} \leq M
$$

for some $j$ such that $f_{j}(x)>f_{j}(y)$, when $x \in X$ and $f_{i}(x)<f_{i}(y)$.

Remark 3.2. Some authors call the efficient solution as Pareto optimal solution. Also, weakly efficient solutions are also called weak-Pareto optimal solution or weak minimum.

Remark 3.3. Kuhn and Tucker noted that some efficient solutions presented an undesirable property with respect to the ratio between the marginal profit of an objective function and the loss of some other functions. To these solutions, they introduced the concept of the noninferior proper solution. Subsequently, Geoffrion [9] modified the concept slightly and defined the properly efficient solutions.

Now, we assume that $f_{i}$ are locally Lipschitz and invex functions on $X$ with respect to $\eta$. We consider in this section the following variational-like inequalities.

Minty-type vectorial variational-like inequality (MVLI). Find $y \in X$ such that for each $x \in X$ and any $\xi_{i} \in \partial f_{i}(x), i=1, \ldots, p$,

$$
\left(\xi_{1}^{T} \eta(x, y), \ldots, \xi_{p}^{T} \eta(x, y)\right) \notin \mathbb{R}_{+}^{n} \backslash\{0\} .
$$

Stampacchia-type vectorial variational-like inequality (SVLI). Find $y \in X$ such that for each $x \in X$ there exist $\xi_{i} \in \partial f_{i}(y), i=1, \ldots, p$, such that

$$
\left(\xi_{1}^{T} \eta(x, y), \ldots, \xi_{p}^{T} \eta(x, y)\right) \notin \mathbb{R}_{+}^{n} \backslash\{0\} .
$$

Proposition 3.4. Assume that $X$ is a nonempty subset of $\mathbb{R}^{n}$ and $f_{i}: \mathbb{R}^{n} \rightarrow \mathbb{R}, i=1, \ldots, p$, are invex locally Lipschitz functions on $X$ with respect to $\eta$. If $y \in X$ is a weakly efficient solution of $(\mathrm{P})$, then it is a solution of MVLI.

Proof. Let $y \in X$ be an efficient solution of (P). Then, for any $x \in X$, we have

$$
f(x)-f(y) \notin-\mathbb{R}_{+}^{n} \backslash\{0\} .
$$

Since $f_{i}$ is invex with respect to $\eta, f_{i}^{0}(x, \eta(y, x)) \leq f_{i}(y)-f_{i}(x)$; therefore,

$$
\xi_{i}^{T} \eta(y, x) \leq f_{i}^{0}(x, \eta(y, x)) \leq f_{i}(y)-f_{i}(x), \quad \forall \xi_{i} \in \partial f_{i}(x) .
$$

From (3.6) and (3.7), we obtain that $y \in X$ is a solution of MVLI. 
Theorem 3.5. Let $X$ be a nonempty subset of $\mathbb{R}^{n}$ and $f_{i}: \mathbb{R}^{n} \rightarrow \mathbb{R}, i=1, \ldots, p$, are invex locally Lipschitz functions on $X$ with respect to $\eta$. If $y \in X$ is a solution of SVLI, then $y$ is an efficient solution of $(\mathrm{P})$.

Proof. Let $y \in X$ be a solution of SVLI. Then for each $x \in X$, there exist $\xi_{i} \in \partial f_{i}(y), i=$ $1, \ldots, p$, such that

$$
\left(\xi_{1}^{T} \eta(x, y), \ldots, \xi_{p}^{T} \eta(x, y)\right) \notin \mathbb{R}_{+}^{n} \backslash\{0\}
$$

Since the functions $f_{i}$ are invex, we have for each $x \in X$,

$$
f_{i}(x)-f_{i}(y) \geq f_{i}^{0}(y, \eta(x, y)) \geq \xi_{i}^{T} \eta(x, y) .
$$

Hence, from (3.8) and (3.9) we obtain

$$
\left(f_{1}(x)-f_{1}(y), \ldots, f_{p}(x)-f_{p}(y)\right) \notin \mathbb{R}_{+}^{n} \backslash\{0\},
$$

and so $y \in X$ is an efficient solution of $(\mathrm{P})$.

From Proposition 3.4 and Theorem 3.5, we have the following corollary.

Corollary 3.6. Let $X$ be a nonempty subset of $\mathbb{R}^{n}$ and $f_{i}: \mathbb{R}^{n} \rightarrow \mathbb{R}, i=1, \ldots, p$, are invex locally Lipschitz functions on $X$ with respect to $\eta$. If $y \in X$ is a solution of SVLI, then $y$ is a solution of MVLI.

Thus, SVLI is a sufficient condition for efficiency in (P). However, this condition is not necessary (see, e.g., [17, page 172]). We will show that it is a necessary condition for proper efficiency in $(\mathrm{P})$. To prove the last assertion we will make use of the following result due to Jeyakumar [15].

Lemma 3.7. Let $\Gamma$ be an arbitrary set, $Y$ a Hausdorff topological vector space, $D$ a compact subset of $Y$, and $F: \Gamma \times D \rightarrow \mathbb{R}$ a function such that $F(x, \cdot)$ is concave and upper semicontinuous on $D$ for each $x \in \Gamma$ fixed and $F(\cdot, y)$ is convex, for each $y \in D$ fixed. Then,

$$
\inf _{x \in \Gamma} \max _{y \in D} F(x, y) \geq 0 \Longleftrightarrow \sup _{y \in D} \inf _{x \in \Gamma} F(x, y) \geq 0
$$

Proposition 3.8. Let $X$ be a compact subset of $\mathbb{R}^{n}$ and $f_{i}: \mathbb{R}^{n} \rightarrow \mathbb{R}, i=1, \ldots, p$, are invex locally Lipschitz functions with respect to $\eta$ on $X$. Consider the following assertions:

(a) $y \in X$ is a properly efficient solution of (P);

(b) there exist $\lambda_{i}>0, i=1, \ldots, p$, such that $y$ is a solution of the following scalar variational-like inequality: find $y \in X$ such that there exists $\xi_{i} \in \partial f_{i}(y), i=1, \ldots, p$, such that for each $x \in X$,

$$
\left(\lambda_{1} \xi_{1}+\cdots+\lambda_{p} \xi_{p}\right)^{T} \eta(x, y) \geq 0
$$


(c) there exist $\lambda_{i}>0, i=1, \ldots, p$, such that $y$ is solution of the following scalar variationallike inequality: find $y \in X$ such that for each $x \in X$, there exists $\xi_{i} \in \partial f_{i}(y), i=$ $1, \ldots, p$, such that

$$
\left(\lambda_{1} \xi_{1}+\cdots+\lambda_{p} \xi_{p}\right)^{T} \eta(x, y) \geq 0
$$

Then (a) and (b) are equivalent, and if $\eta(\cdot, y)$ is linear for each $y \in X$, then (a), (b), and (c) are equivalent.

Proof. (a) $\Rightarrow$ (b). If $y \in X$ is a properly efficient solution of $(\mathrm{P})$. It follows from the invexity of the functions $f_{i}$ on $X$ that there exist $\lambda_{i}>0, i=1, \ldots, p$, (see Brandão et al. [3]) such that $y \in X$ is a solution of the scalar optimization problem:

$$
\text { minimize } \lambda_{1} f_{1}(x)+\cdots+\lambda_{p} f_{p}(x) \quad \text { subject to } x \in X \text {. }
$$

We observe that the function $\lambda_{1} f_{1}+\cdots+\lambda_{p} f_{p}$ is invex and thus

$$
0 \in \partial\left(\sum_{i=1}^{p} \lambda_{i} f_{i}\right)(y)+N_{X}(y) \subset \sum_{i=1}^{p} \lambda_{i} \partial f_{i}(y)+N_{X}(y)
$$

Then, there exist $\mu \in N_{X}(y)$ and $\xi_{i} \in \partial f_{i}(y), i=1, \ldots, p$, such that

$$
0=\mu+\sum_{i=1}^{p} \lambda_{i} \xi_{i}
$$

On the other hand, $\eta(x, y) \in T_{X}(y)$, for all $x \in X$ and, furthermore,

$$
\langle\mu, \eta(x, y)\rangle \leq 0, \quad \forall x \in X
$$

From (3.15) and (3.16) follows $\sum_{i=1}^{p} \lambda_{i}\left\langle\xi_{i}, \eta(x, y)\right\rangle \geq 0$, for all $x \in X$, that is,

$$
\left(\lambda_{1} \xi_{1}+\cdots+\lambda_{p} \xi_{p}\right)^{T} \eta(x, y) \geq 0 .
$$

Hence, (b) is proved.

(b) $\Rightarrow(\mathrm{a})$. We assume that there exists $y \in X$ such that $\xi_{i} \in \partial f_{i}(y), i=1, \ldots, p$, such that for each $x \in X\left(\lambda_{1} \xi_{1}+\cdots+\lambda_{p} \xi_{p}\right)^{T} \eta(x, y) \geq 0$ is verified. We will assume that it is not a properly efficient solution of $(\mathrm{P})$ and then exhibiting a contradiction. Suppose $y$ is not a solution of the following scalar minimization problem (see [9]):

$$
\text { minimize } \lambda_{1} f_{1}(x)+\cdots+\lambda_{p} f_{p}(x), \quad \text { subject to } x \in X
$$

that is, there exists $x \in X$ such that $\sum_{i=1}^{p} \lambda_{i} f_{i}(x)<\sum_{i=1}^{p} \lambda_{i} f_{i}(y)$ and, furthermore,

$$
\sum_{i=1}^{p} \lambda_{i}\left(f_{i}(x)-f_{i}(y)\right)<0
$$


Now, using the invexity of the functions $f_{i}$, we obtain

$$
\sum_{i=1}^{p} \lambda_{i}\left(f_{i}(x)-f_{i}(y)\right) \geq \sum_{i=1}^{p} \lambda_{i} f_{i}^{0}(y, \eta(x, y)) \geq \sum_{i=1}^{p} \lambda_{i} \xi_{i}^{T} \eta(x, y) \geq 0
$$

which contradicts (3.18). Hence, $y$ is a properly efficient solution of $(\mathrm{P})$.

(b) $\Leftrightarrow(\mathrm{c})$. We suppose that $y \in X$ and that there exist $\lambda_{i}>0$ such that there are $\xi_{i} \in$ $\partial f_{i}(y), i=1, \ldots, p$, such that, for each $x \in X,\left(\lambda_{1} \xi_{1}+\cdots+\lambda_{p} \xi_{p}\right)^{T} \eta(x, y) \geq 0$ is verified. Equivalently,

$$
\max _{\xi_{i} \in \partial f_{i}(y)} \inf _{x \in X}\left(\lambda_{1} \xi_{1}+\cdots+\lambda_{p} \xi_{p}\right)^{T} \eta(x, y) \geq 0
$$

Define

$$
\begin{gathered}
D:=\prod_{i=1}^{p} \partial f_{i}(y), \\
\Gamma:=X, \\
F(x, \xi):=\left(\lambda_{1} \xi_{1}+\cdots+\lambda_{p} \xi_{p}\right)^{T} \eta(x, y) .
\end{gathered}
$$

The function $F$ satisfies the hypotheses of Lemma 3.7. In fact, for $x \in \Gamma$ fixed, $F(x, \cdot)$ is continuous on $D$ (because it is a linear form defined between finite-dimensional spaces and, in particular, is upper semicontinuous). Moreover, $F(x, \cdot)$ is simultaneously concave and convex. Consequently, (3.21) is equivalent to

$$
\inf _{x \in X} \max _{\xi_{i} \in \partial f_{i}(y)}\left(\lambda_{1} \xi+\cdots+\lambda_{p} \xi_{p}\right)^{T} \eta(x, y) \geq 0
$$

and this last inequality is exactly the statement (b). Thus, (b) and (c) are equivalent.

From Proposition 3.8, we get the following theorem.

Theorem 3.9. Let $X$ be a compact and nonempty subset of $\mathbb{R}^{n}$ and $f_{i}: \mathbb{R}^{n} \rightarrow \mathbb{R}, i=1, \ldots, p$, are invex locally Lipschitz functions on $X$ with respect to $\eta$. If $y$ is a properly efficient solution of $(\mathrm{P})$, then $y$ is a solution of SVLI.

Proof. If $y$ is a properly efficient solution of $(\mathrm{P})$, then using Proposition 3.8, there exist $\lambda_{i}>0, \xi \in \partial f_{i}(y), i=1, \ldots, p$, such that

$$
\left(\lambda_{1} \xi+\cdots+\lambda_{p} \xi_{p}\right)^{T} \eta(x, y) \geq 0, \quad \forall x \in X .
$$

In fact, assume that there exists $x \in X$ such that for each $\xi_{i} \in \partial f_{i}(y)$, we have

$$
\left(\xi_{1}^{T} \eta(x, y), \ldots, \xi_{p}^{T} \eta(x, y)\right) \in-\mathbb{R}_{+}^{p} \backslash\{0\}
$$


and we show a contradiction. Since $\lambda_{i}$ are all strictly positive, we conclude

$$
\sum_{i=1}^{p}\left(\lambda_{i} \xi_{i}\right)^{T} \eta(x, y)<0,
$$

which contradicts (3.24). Hence, $y$ is solution of SVLI.

\section{Weakly efficient solutions and variational-like inequalities}

In this section, we will consider variational-like inequalities of weak Minty and weak Stampacchia types, which we formulate as follows.

Weak Minty variational-like inequality (WMVLI). Find $y \in X$ such that, for each $x \in X$ and each $\xi_{i} \in \partial f_{i}(x), i=1, \ldots, p$, we have

$$
\left(\xi_{1}^{T} \eta(x, y), \ldots, \xi_{p}^{T} \eta(x, y)\right) \notin-\operatorname{int} \mathbb{R}_{+}^{p} .
$$

Weak Stampacchia variational-like inequality (WSVLI). Find $y \in X$ such that for each $x \in X$, there exist $\xi_{i} \in \partial f_{i}(y), i=1, \ldots, p$, such that

$$
\left(\xi_{1}^{T} \eta(x, y), \ldots, \xi_{p}^{T} \eta(x, y)\right) \notin-\operatorname{int} \mathbb{R}_{+}^{p} .
$$

Under certain hypotheses, it is possible to show that the solutions of WMVLI and WSVLI are equals. Before, we prove this, will recall the following definition: given the function $\eta: S \times S \rightarrow \mathbb{R}^{n}$ where $S$ is a nonempty subset of $\mathbb{R}^{n}$, we say that $S$ is invex with respect to $\eta$ at $x \in S$ if for each $y \in S$ and each $t \in[0,1]$, we have $x+t \eta(y, x) \in S$; we say that $S$ is invex if it is invex for all $x \in S$.

Theorem 4.1. Let $X$ be a nonempty subset of $\mathbb{R}^{n}$, invex with respect to $\eta$ and $f_{i}: \mathbb{R}^{n} \rightarrow \mathbb{R}$, $i=1, \ldots, p$, are invex locally Lipschitz functions with respect to $\eta$.

(1) If $y$ is a solution of WMVLI, then $y$ is a solution of WSVLI.

(2) Assume that the function $\eta$ is antisymmetric (i.e., $\eta(x, y)=-\eta(y, x), \forall x, y \in X)$ and that $y \in X$ is a solution of WSVLI. Then $y$ is a solution of WMVLI.

Proof. (1) We suppose that $y \in X$ is solution of WSVLI. Then, for each $x \in X$, there are $\xi_{i} \in \partial f_{i}(y), i=1, \ldots, p$ such that

$$
\left(\xi_{1}^{T} \eta(x, y), \ldots, \xi_{p}^{T} \eta(x, y)\right) \notin-\operatorname{int} \mathbb{R}_{+}^{p} .
$$

Let $\hat{\xi}_{i} \in \partial f_{i}(x), i=1, \ldots, p$. We claim that $\left(\xi_{i}-\widehat{\xi}_{i}\right)^{T} \eta(x, y) \leq 0, i=1, \ldots, p$. In fact, from the antisymmetry of $\eta$ and (4.3), we have

$$
\left(\xi_{i}-\widehat{\xi}_{i}\right)^{T} \eta(x, y)=\xi_{i} \eta(x, y)-\widehat{\xi}_{i} \eta(x, y)=\xi_{i} \eta(x, y)+\widehat{\xi}_{i} \eta(y, x) .
$$

Also, since $f_{i}$ is invex,

$$
\begin{aligned}
& \xi_{i}^{T} \eta(x, y) \leq f_{i}^{\prime}(y, \eta(x, y)) \leq f_{i}(x)-f_{i}(y), \quad i=1, \ldots, p, \\
& \hat{\xi}_{i}^{T} \eta(y, x) \leq f_{i}^{0}(x, \eta(y, x)) \leq f_{i}(y)-f_{i}(x), \quad i=1, \ldots, p,
\end{aligned}
$$


and adding (4.5), we obtain

$$
\left(\xi_{i}-\widehat{\xi}_{i}\right)^{T} \eta(x, y) \leq 0, \quad i=1, \ldots, p,
$$

that is, for each $x \in X$ and each $\hat{\xi}_{i} \in \partial f_{i}(x), i=1, \ldots, p$, we have

$$
\left(\hat{\xi}_{1}^{T} \eta(x, y), \ldots, \hat{\xi}_{p}^{T} \eta(x, y)\right) \geq\left(\xi_{1}^{T} \eta(x, y), \ldots, \xi_{p}^{T} \eta(x, y)\right) \notin-\operatorname{int} \mathbb{R}_{+}^{p}
$$

and, consequently,

$$
\left(\hat{\xi}_{1}^{T} \eta(x, y), \ldots, \hat{\xi}_{p}^{T} \eta(x, y)\right) \notin-\operatorname{int} \mathbb{R}_{+}^{p} ;
$$

thus, $y$ is a solution of WMVLI.

(2) We will prove the statement (2). For that, we suppose that $y \in X$ is a solution of WMVL. In this case, for each $x \in X$ and each $\xi_{i} \in \partial f_{i}(x), i=1, \ldots, p$, we have $\left(\xi_{1}^{T} \eta(x, y)\right.$, $\left.\ldots, \xi_{p}^{T} \eta(x, y)\right) \notin-$ int $\mathbb{R}_{+}^{p}$. For $z \in X$ fixed, we consider the sequence $\left(\alpha_{k}\right) \subset(0,1]$, with $\alpha_{k} \rightarrow 0$ when $k \rightarrow \infty$ and we define $z_{k}:=y+\alpha_{k} \eta(z, y)$. Since $X$ is invex with respect to $\eta$, then the sequence $\left(z_{k}\right)$ belongs to $X$. The set $\partial f_{i}\left(z_{k}\right)$ is nonempty and therefore we can take $\xi_{i}^{k} \in \partial f_{i}\left(z_{k}\right), i=1, \ldots, p$, for each $k \in \mathbb{N}$. But $y$ is a solution of WMVLI and therefore

$$
\left(\xi_{1}^{k T} \eta(x, y), \ldots, \xi_{p}^{k T} \eta(x, y)\right) \notin-\operatorname{int} \mathbb{R}_{+}^{p} .
$$

Without loss of generality, we can suppose that all the functions $f_{i}$ have the same Lipschitz constant $K$. Since $\xi_{i}^{k} \in \partial f_{i}\left(z_{k}\right)$, for each $k$, we have $\left\|\xi_{i}^{k}\right\| \leq K$, for $i=1, \ldots, p$. For each $i,\left(\xi_{i}^{k}\right)_{k \in \mathbb{N}}$ is a bounded sequence in $\mathbb{R}^{n}$ and we can assume that $\xi_{i}^{k} \rightarrow \hat{\xi}_{i}$ when $k \rightarrow \infty$, for some $\hat{\xi}_{i} \in \mathbb{R}^{n}$. Also, $\xi_{i}^{k} \in \partial f_{i}\left(z_{k}\right)$ for each $k$ and $z_{k} \rightarrow y$ and since the set-valued mapping $\partial f_{i}$ is closed (see [6]), we obtain $\hat{\xi}_{i} \in \partial f_{i}(y), i=1, \ldots, p$. Taking $k \rightarrow \infty$ in (4.9) and observing that the set $\left(- \text { int } \mathbb{R}_{+}^{p}\right)^{c}$ is closed in $\mathbb{R}^{p}$, we obtain

$$
\left(\hat{\xi}_{1}^{T} \eta(x, y), \ldots, \hat{\xi}_{p}^{T} \eta(x, y)\right) \notin-\operatorname{int} \mathbb{R}_{+}^{p}
$$

Hence, $y \in X$ is a solution of WSVLI.

Theorem 4.2. Let $X$ be a nonempty subset of $\mathbb{R}^{n}$, invex with respect to $\eta$ and $f_{i}: \mathbb{R}^{n} \rightarrow \mathbb{R}$, $i=1, \ldots, p$, invex locally Lipschitz functions with respect to $\eta$ on $X$. Then

(1) if $y \in X$ is a solution of WSVLI, then $y$ is a weakly efficient solution of $(\mathrm{P})$;

(2) if $\eta$ is antisymmetric, then $y \in X$ is a weakly efficient solution of $(\mathrm{P})$ if and only if $y$ is a solution of WSVLI.

Proof. Initially suppose that $y \in X$ is not a weakly efficient solution of (P). Then there exists $z \in X$ such that

$$
f_{i}(y)>f_{i}(z), \quad i=1, \ldots, p .
$$


Let $\xi_{i} \in \partial f_{i}(y), i=1, \ldots, p$. Since the $f_{i}$ are invex, we have

$$
\xi_{i}^{T} \eta(z, y) \leq f_{i}^{0}(y, \eta(z, y)) \leq f_{i}(z)-f_{i}(y) .
$$

Thus,

$$
f_{i}(z)-f_{i}(y) \geq \xi_{i}^{T} \eta(z, y), \quad \forall \xi_{i} \in \partial f_{i}(y)
$$

and, from (4.11) and (4.13), we have

$$
\left(\xi_{1}^{T} \eta(z, y), \ldots, \xi_{p}^{T} \eta(z, y)\right) \in-\operatorname{int} \mathbb{R}_{+}^{P} .
$$

Consequently, $y$ is not a solution of WSVLI.

Now, we suppose that $y \in X$ is not solution of WSVLI. In this case, there exists $\bar{x} \in X$ such that for each $\xi_{i} \in \partial f_{i}(\bar{x}), i=1, \ldots, p$, we have

$$
\left(\xi_{1}^{T} \eta(\bar{x}, y), \ldots, \xi_{p}^{T} \eta(\bar{x}, y)\right) \in-\operatorname{int} \mathbb{R}_{+}^{p} .
$$

Let $\xi_{i} \in \partial f_{i}(\bar{x})$. Since the $f_{i}$ are invex,

$$
f_{i}(y)-f_{i}(\bar{x}) \geq f_{i}^{0}(\bar{x}, \eta(y, \bar{x})) \geq \xi_{i}^{T} \eta(y, \bar{x}), \quad i=1, \ldots, p,
$$

and consequently

$$
\xi_{i}^{T} \eta(y, \bar{x}) \geq f_{i}(y)-f_{i}(\bar{x})
$$

The function $\eta$ is antisymmetric and from (4.17), we have

$$
\xi_{i}^{T} \eta(\bar{x}, y) \geq f_{i}(\bar{x})-f_{i}(y), \quad i=1, \ldots, p .
$$

Thus $y$ is not a weakly efficient solution of $(\mathrm{P})$.

Next, we will show results as in Proposition 3.8 for a weakly efficient solution of $(\mathrm{P})$. To do this, we will use the following result that is an alternative theorem for invex functions, its proof can be seen in [3].

Lemma 4.3 (invex Gordan's theorem). Let $C$ be a nonempty closed subset of $\Omega$. Suppose that $f_{i}: \Omega \rightarrow \mathbb{R}, i \in I=\{1, \ldots, m\}$, are locally Lipschitz functions and invex on $C$, for a common $\eta$. If $f(x)=\max \left\{f_{i}(x): i \in I\right\}$ reaches a minimum on $C$, then either

(i) there exists $x \in C$ such that $f_{i}(x)<0$, for all $i \in I$;

(ii) there exist $\lambda_{i} \geq 0, i \in I$, not all zero, such that $\sum_{i \in I} \lambda_{i} f_{i}(x) \geq 0$, for all $x \in C$;

but never both.

Naturally, under the hypotheses of Lemma 4.3, if $C$ is compact, then $f(x)$ reaches a minimum on $C$.

Next, we state the following result.

Proposition 4.4. Let $X$ be a compact, nonempty subset of $\mathbb{R}^{n}$ and $f_{i}: \mathbb{R}^{n} \rightarrow \mathbb{R}$ are invex locally Lipschitz functions with respect to $\eta$ on $X$. Assume that for each $y \in X$, the function 
$\eta(\cdot, y)$ is linear. Then, the following statements are equivalent:

(a) $y \in X$ is a weakly efficient solution of $(\mathrm{P})$;

(b) there exist $\lambda_{i} \geq 0, i=1, \ldots, p$, not all zero, such that $y \in X$ is a solution of the following scalar variational-like inequality: find $y \in X$ such that there exist $\xi_{i} \in \partial f_{i}(y), i=$ $1, \ldots, p$, such that, for each $x \in X$,

$$
\left(\lambda_{1} \xi_{1}+\cdots+\lambda_{p} \xi_{p}\right)^{T} \eta(x, y) \geq 0 ;
$$

(c) there exist $\lambda_{i} \geq 0, i=1, \ldots, p$, not all zero, such that $y \in X$ is a solution of the following scalar variational-like inequality: find $y \in X$ such that for each $x \in X$, there exist $\xi_{i} \in \partial f_{i}(y)$ such that

$$
\left(\lambda_{1} \xi_{1}+\cdots+\lambda_{p} \xi_{p}\right)^{T} \eta(x, y) \geq 0 .
$$

Proof. The equivalence between (b) and (c) is proved similarly as in Proposition 3.8.

(a) $\Rightarrow(\mathrm{b})$. We assume that $y \in X$ is a weakly efficient solution of $(\mathrm{P})$. Since $f_{i}$ are invex, the functions $\phi_{i}$,

$$
\phi_{i}(x)=f_{i}(x)-f_{i}(y), \quad x \in X
$$

are also invex with respect to $\eta$ on $X$. By hypotheses, it does not exist an $x \in X$ such that $\phi_{i}(x)<0, i=1, \ldots, p$. Consequently, from Lemma 4.3 , there exist $\lambda_{i} \geq 0, i=1, \ldots, p$, not all zero and such that $\sum_{i=1}^{p} \lambda_{i} \phi_{i} \geq 0$, for all $x \in X$. Or equivalently, $y$ is a solution of the scalar problem (SP'):

$$
\operatorname{minimize} \sum_{i=1}^{p} \lambda_{i} f_{i}(x) \quad \text { subject to } x \in X
$$

and hence

$$
0 \in \partial\left(\sum_{i=1}^{p} \lambda_{i} f_{i}\right)(y)+N_{X}(y) \subset \sum_{i=1}^{p} \partial f_{i}(y)+N_{X}(y) .
$$

Thus, there exist $\mu \in N_{X}(y), \xi_{i} \in \partial f_{i}(y), i=1, \ldots, p$, such that $0=\mu+\sum_{i=1}^{p} \lambda_{i} \xi_{i}$. Since $\eta(x, y) \in T_{X}(y)$, for all $x \in X$, we obtain

$$
0 \geq\langle\mu, \eta(x, y)\rangle=-\sum_{i=1}^{p} \lambda_{i}\left\langle\xi_{i}, \eta(x, y)\right\rangle, \quad \forall x \in X,
$$

that is,

$$
\left(\lambda_{1} \xi_{1}+\cdots+\lambda_{p} \xi_{p}\right)^{T} \eta(x, y) \geq 0 ;
$$

that is exactly the statement (b).

(b) $\Rightarrow(\mathrm{a})$. We assume that there exist $\lambda_{i} \geq 0, i=1, \ldots, p$, not all zero, $\xi_{i} \in \partial f_{i}(y)$ such that

$$
\left(\lambda_{1} \xi_{1}+\cdots+\lambda_{p} \xi_{p}\right)^{T} \eta(x, y) \geq 0, \quad \forall x \in X
$$


If $y$ is not a weakly efficient solution of (P), then $y$ is not a solution of the weighted scalar problem associated to $\lambda$, that is, there exists $x \in X$ such that $\sum_{i=1}^{p} \lambda_{i}\left(f_{i}(x)-f_{i}(y)\right)<0$. On the other hand,

$$
\begin{aligned}
0 & >\sum_{i=1}^{p} \lambda_{i}\left(f_{i}(x)-f_{i}(y)\right) \geq \sum_{i=1}^{p} \lambda_{i} f_{i}^{0}(y, \eta(x, y)) \geq \sum_{i=1}^{p} \xi_{i}^{T} \eta(x, y) \\
& =\left(\lambda_{1} \xi_{1}+\cdots+\lambda_{p} \xi_{p}\right)^{T} \eta(x, y) \geq 0
\end{aligned}
$$

which is absurd.

From Theorems 4.1 and 4.2 and Proposition 4.4, we easily obtain the following theorem.

TheOREM 4.5. Let $X$ be a compact, nonempty subset of $\mathbb{R}^{n}$, invex with respect to $\eta$, and $f_{i}: \mathbb{R}^{n} \rightarrow \mathbb{R}, i=1, \ldots, p$, are invex locally Lipschitz functions with respect to $\eta$ on $X$. Let the function $\eta$ be antisymmetric and such that $\eta(\cdot, y)$ is linear for each $y \in Y$. Then, the following assertions are equivalent:

(1) $y \in X$ is a weakly efficient solution of $(\mathrm{P})$;

(2) $y \in X$ is a solution of WMVLI;

(3) $y \in X$ is a solution of WSVLI;

(4) there exist $\lambda_{i} \geq 0, i=1, \ldots, p$, not all zero, and such that $y \in X$ is a solution of the following scalar variational-like inequality: to find $y \in X$ such that there exist $\xi_{i} \in$ $\partial f_{i}(y), i=1, \ldots, p$, such that for each $x \in X$,

$$
\left(\lambda_{1} \xi_{1}+\cdots+\lambda_{p} \xi_{p}\right)^{T} \eta(x, y) \geq 0
$$

(5) there exist $\lambda_{i} \geq 0, i=1, \ldots, p$, not all zero, and such that $y \in X$ is a solution of the following scalar variational-like inequality: to find $y \in X$ such that for each $x \in X$, there exist $\xi_{i} \in \partial f_{i}(y), i=1, \ldots, p$, such that

$$
\left(\lambda_{1} \xi_{1}+\cdots+\lambda_{p} \xi_{p}\right)^{T} \eta(x, y) \geq 0 .
$$

Note that the hypotheses applied on $\eta$ in the last theorem are trivially satisfied when the function $f_{i}$ are convex; in effect, in this case, $\eta(x, y)=x-y$. See [24].

\section{Existence of weakly efficient solutions}

We begin this section by recalling a fixed point theorem for set-valued mappings which is a generalization of the classical fixed point theorem of Fan-Browder, and which proof can be found in Park [21].

Lemma 5.1 (fixed point theorem). Let $X$ be a convex, nonempty subset of a Hausdorff topological vector space $E$ and $K$ a compact nonempty subset of $X$. Let $A$ and $B: X \rightrightarrows X$ be two set-valued mappings satisfying the following conditions:

(1) $A x \subset B x$, for all $x \in X$;

(2) $B x$ is a convex set, for all $x \in X$;

(3) $A x \neq \varnothing$, for all $x \in K$; 
(4) $A^{-1} y=\{x \in X: y \in A x\}$ is an open set, for all $y \in X$;

(5) for each $N$ finite subset of $X$, there exists $L_{N}$ compact, convex, nonempty subset of $X$ such that $L_{N} \supset N$ and for each $x \in L_{N} \backslash K, A x \cap L_{N} \neq \varnothing$.

Then, there is a $\bar{x} \in X$ such that $\bar{x} \in B \bar{x}$.

We will use the results of the previous section, together with Lemma 5.1, to establish a result about the existence of weakly efficient solution for the nonsmooth invex vectorial problems under weak compactness hypothesis on the feasible set $X$.

Theorem 5.2. Let $X$ be a nonempty subset of $\mathbb{R}^{n}$, invex with respect to $\eta$ and $f_{i}: \mathbb{R}^{n} \rightarrow \mathbb{R}$, $i=1, \ldots, p$, are invex locally Lipschitz functions with respect to $\eta$. Assume that $\eta$ is an antisymmetric function and such that $\eta(\cdot, y)$ is convex and continuous, for each $y \in X$. Suppose also that there exists a compact, nonempty subset $K$ of $X$ such that for each finite subset $N$ of $X$, there exists a compact, convex, and nonempty subset $L_{N}$ of $X$, such that $L_{N} \supset N$ and for all $x \in L_{N} \backslash K$, there is $z \in L_{N}$ such that there exist $\xi_{i} \in \partial f_{i}(z), i=1, \ldots, p$, satisfying

$$
\left(\xi_{1}^{T} \eta(z, x), \ldots, \xi_{p}^{T} \eta(z, x)\right) \in-\operatorname{int} \mathbb{R}_{+}^{p} .
$$

Then, (P) has a weakly efficient solution.

Proof. We use a concise notation to make the proof clearer. We denote by $\partial f(x)$ the set $\partial f_{1}(x) \times \cdots \times \partial f_{p}(x), x \in X$. Let $s=\left(s_{1}, \ldots, s_{p}\right)$, where $s_{i} \in \mathbb{R}^{n}, i=1, \ldots, p$. Let $s^{T} \eta(x, y)$ be the vector

$$
\left(s_{1}^{T} \eta(x, y), \ldots, s_{p}^{T} \eta(x, y)\right) \in \mathbb{R}^{p} .
$$

Let $A$ and $B: X \rightrightarrows X$ be two set-valued mappings given by

$$
\begin{aligned}
& A x:=\left\{z \in X: \exists t \in \partial f(z), t^{T} \eta(z, x) \in-\operatorname{int} \mathbb{R}_{+}^{p}\right\}, \\
& B x:=\left\{z \in X: \forall s \in \partial f(x), s^{T} \eta(z, x) \in-\operatorname{int} \mathbb{R}_{+}^{p}\right\} .
\end{aligned}
$$

We will prove (using Lemma 5.1) that there exists $y \in K$ such that $A y=\varnothing$ or, equivalently, $y$ is a solution of WMVLI and by Theorem 4.2, it is sufficient to prove our result. Initially, we will prove that the set-valued mappings $A$ and $B$ satisfy the conditions (1), (2), (4), and (5) of Lemma 5.1 and that $B$ does not have a fixed point. So, Lemma 5.1 will imply the existence of $y \in K$ such that $A y=\varnothing$.

Next, we will show that the condition (1) of Lemma 5.1 holds: let $x \in X$ and $z \in A x$. Then there exist $t=\left(\xi_{1}, \ldots, \xi_{p}\right) \in \partial f(z)$ such that

$$
\left(\xi_{1}^{T} \eta(z, x), \ldots, \xi_{p}^{T} \eta(z, x)\right) \in-\operatorname{int} \mathbb{R}_{+}^{p} .
$$

Let $s=\left(\hat{\xi}_{1}, \ldots, \hat{\xi}_{p}\right) \in \partial f(x)$. Using the invexity of functions $f_{i}$ and the antisymmetry of $\eta$, we have, for each $i=1, \ldots, p$,

$$
\begin{aligned}
\hat{\xi}_{i} \eta(z, x) & \leq f_{i}^{0}(x, \eta(z, x)) \leq f_{i}(z)-f_{i}(x)=-\left(f_{i}(x)-f_{i}(z)\right) \\
& \leq-\xi_{i}^{T} \eta(x, z)=\xi_{i}^{T} \eta(z, x) .
\end{aligned}
$$


From (5.4) and (5.5), we obtain

$$
\left(\widehat{\xi_{1}^{T}} \eta(z, x), \ldots, \widehat{\xi_{p}^{T}} \eta(z, x)\right) \in-\operatorname{int} \mathbb{R}_{+}^{p}
$$

where $z \in B x$.

Now, we will see that the second condition of Lemma 5.1 holds. Let $x \in X, z_{1}, z_{2} \in B x$, and $\lambda \in[0,1]$. Then, for each $s=\left(\xi_{1}, \ldots, \xi_{p}\right) \in \partial f(x)$, we have

$$
\left(\xi_{1}^{T} \eta\left(z_{1}, x\right), \ldots, \xi_{p}^{T} \eta\left(z_{1}, x\right)\right),\left(\xi_{1}^{T} \eta\left(z_{2}, x\right), \ldots, \xi_{p}^{T} \eta\left(z_{2}, x\right)\right) \in-\operatorname{int} \mathbb{R}_{+}^{p}
$$

For each $j=1, \ldots, p$, we consider $\xi_{j}=\left(\xi_{j}^{(1)}, \ldots, \xi_{j}^{(n)}\right), \xi_{j}^{(k)} \in \mathbb{R}, \eta(x, y)=\left(\eta_{1}(x, y), \ldots\right.$, $\left.\eta_{n}(x, y)\right), \eta_{k}(x, y) \in \mathbb{R}$. Then, from the convexity of $\eta_{k}$ and (5.7), we obtain

$$
\begin{aligned}
\xi_{j}^{T} \eta\left(\lambda z_{1}+(1-\lambda) z_{2}, x\right) & =\sum_{k=1}^{n} \xi_{j}^{(k)} \eta_{k}\left(\lambda z_{1}+(1-\lambda) z_{2}, x\right) \\
& \leq \sum_{k=1}^{n} \xi_{j}^{(k)}\left[\lambda \eta_{k}\left(z_{1}, x\right)+(1-\lambda) \eta_{k}\left(z_{2}, x\right)\right] \\
& =\lambda \xi_{j}^{T} \eta\left(z_{1}, x\right)+(1-\lambda) \xi_{j}^{T} \eta\left(z_{2}, x\right)<0, \quad j=1, \ldots, p .
\end{aligned}
$$

Hence, $\lambda z_{1}+(1-\lambda) z_{2} \in B x$.

The fourth condition is proved as follows: we prove that, for all $z \in X$, the set $\left(A^{-1} z\right)^{c}$ is closed. To do this, we consider a sequence $\left(x_{n}\right) \subset\left(A^{-1} z\right)^{c}$ and such that $x_{n}$ converges to $x$. Then, $x_{n} \notin A^{-1} z$, for all $n \in \mathbb{N}$. Let $t=\left(\xi_{1}, \ldots, \xi_{p}\right) \in \partial f(z)$, such that

$$
\left(\xi_{1}^{T} \eta\left(z, x_{n}\right), \ldots, \xi_{p}^{T} \eta\left(z, x_{n}\right)\right) \notin-\operatorname{int} \mathbb{R}_{+}^{p}
$$

Since $\eta(\cdot, z)$ is continuous and antisymmetric, we have that $\eta(z, \cdot)$ is also continuous and antisymmetric; $\left(-\operatorname{int} \mathbb{R}_{+}^{p}\right)^{c}$ being closed, taking $n \rightarrow \infty$ in (5.9), we obtain

$$
\left(\xi_{1}^{T} \eta(z, x), \ldots, \xi_{p}^{T} \eta(z, x)\right) \notin-\operatorname{int} \mathbb{R}_{+}^{p}
$$

and thus $x \in\left(A^{-1} z\right)^{c}$.

By our hypotheses, condition (5) of Lemma 5.1 holds.

However, $B$ does not have a fixed point, because if there is a fixed point, it would exist some $x \in X$ such that for each $s \in \partial f(x), s^{T} \eta(x, x)=0 \in-\operatorname{int} \mathbb{R}_{+}^{p}$, which is absurd.

Consequently, from Lemma 5.1, there exists $y \in K$ such that $A y=\varnothing$.

Corollary 5.3. Let $X$ be a nonempty subset of $\mathbb{R}^{n}$, invex with respect to $\eta$ and $\eta$ antisymmetric such that $\eta(\cdot, y)$ is convex and continuous. If

$$
K=\left\{x \in X:\left(f_{1}^{0}\left(z_{0}, \eta\left(z_{0}, x\right)\right), \ldots, f_{p}^{0}\left(z_{0}, \eta\left(z_{0}, x\right)\right)\right) \notin-\operatorname{int} \mathbb{R}_{+}^{p}\right\}
$$

is compact for some $z_{0} \in X$. Then, (P) has a weakly efficient solution. 
Proof. Let $N$ be a nonempty and finite subset of $X$. We define $L_{N}:=\overline{\mathrm{co}}(N \cup K)$ (where $\overline{\mathrm{co}}$ denotes the closed convex hull of $A$ ). Then, for each $x \in L_{N} \backslash K \subset X \backslash K$, we have

$$
\left(f_{1}^{0}\left(z_{0}, \eta\left(z_{0}, x\right)\right), \ldots, f_{p}^{0}\left(z_{0}, \eta\left(z_{0}, x\right)\right)\right) \in-\operatorname{int} \mathbb{R}_{+}^{p} .
$$

Let $z:=z_{0} \in K \subset L_{N}, \xi_{i} \in \partial f_{i}(z)$, we have

$$
\xi_{i}^{T} \eta\left(z_{0}, x\right) \leq f_{i}^{0}\left(z_{0}, \eta\left(z_{0}, x\right)\right), \quad i=1, \ldots, p .
$$

Thus, from (5.12) and (5.13), we obtain

$$
\left(\xi_{1}^{T} \eta(z, x), \ldots, \xi_{p}^{T} \eta(z, x)\right) \in-\operatorname{int} \mathbb{R}_{+}^{p} .
$$

Therefore, the hypotheses of Theorem 5.2 are verified and, consequently, (P) has a weakly efficient solution.

\section{Conclusions}

In this paper, we studied the equivalence between solutions of vectorial variational-like inequalities of (weak) Minty and Stampacchia type and the (weak) efficient solutions of the nonsmooth invex vectorial optimization problem. We used an approach analogous to those used by Giannessi [11], generalizing the results proved by Lee in [17].

\section{Acknowledgments}

The authors express their sincere gratitude to the referee for useful remarks which led to the clarification and improvement of several points in the paper. The second author is partially supported by CNPq-Brazil, Grant 301351/03-00 and Grant BFM2003-06579 of the DGES (Spain). The third author is partially supported by Grant BFM2003-06579 of the DGES (Spain).

\section{References}

[1] C. Baiocchi and A. Capelo, Variational and Quasivariational Inequalities: Applications to Free Boundary Problems, A Wiley-Interscience Publication, John Wiley \& Sons, New York, 1984.

[2] A. Ben-Israel and B. Mond, What is invexity?, Journal of the Australian Mathematical Society. Series B 28 (1986), no. 1, 1-9.

[3] A. J. V. Brandão, M. A. Rojas-Medar, and G. N. Silva, Invex nonsmooth alternative theorem and applications, Optimization 48 (2000), no. 2, 239-253.

[4] G. Y. Chen and B. D. Craven, Existence and continuity of solutions for vector optimization, Journal of Optimization Theory and Applications 81 (1994), no. 3, 459-468.

[5] G. Y. Chen and S. J. Li, Existence of solutions for a generalized vector quasivariational inequality, Journal of Optimization Theory and Applications 90 (1996), no. 2, 321-334.

[6] F. H. Clarke, Optimization and Nonsmooth Analysis, Canadian Mathematical Society Series of Monographs and Advanced Texts, John Wiley \& Sons, New York, 1983.

[7] B. D. Craven and B. M. Glover, Invex functions and duality, Journal of the Australian Mathematical Society. Series A 39 (1985), no. 1, 1-20.

[8] G. Duvaut and J.-L. Lions, Les inéquations en Mécanique et en Physique, Dunod, Paris, 1972.

[9] A. M. Geoffrion, Proper efficiency and the theory of vector maximization, Journal of Mathematical Analysis and Applications 22 (1968), no. 3, 618-630. 
[10] F. Giannessi, Theorems of alternative, quadratic programs and complementarity problems, Variational Inequalities and Complementarity Problems (Proc. Internat. School, Erice, 1978) (R. W. Cottle, F. Giannessi, and J.-L. Lions, eds.), Wiley, Chichester, 1980, pp. 151-186.

[11] _ On Minty variational principle, New Trends in Mathematical Programming, Appl. Optim., vol. 13, Kluwer Academic, Massachusetts, 1998, pp. 93-99.

[12] G. Giorgi and A. Guerraggio, Various types of nonsmooth invex functions, Journal of Information \& Optimization Sciences 17 (1996), no. 1, 137-150.

[13] R. Glowinski, J.-L. Lions, and R. Trémolières, Numerical Analysis of Variational Inequalities, North-Holland, Amsterdam, 1976.

[14] M. A. Hanson, On sufficiency of the Kuhn-Tucker conditions, Journal of Mathematical Analysis and Applications 80 (1981), no. 2, 545-550.

[15] V. Jeyakumar, A generalization of a minimax theorem of Fan via a theorem of the alternative, Journal of Optimization Theory and Applications 48 (1986), no. 3, 525-533.

[16] I. V. Konnov and J. C. Yao, On the generalized vector variational inequality problem, Journal of Mathematical Analysis and Applications 206 (1997), no. 1, 42-58.

[17] G. M. Lee, On relations between vector variational inequality and vector optimization problem, Progress in Optimization (Perth, 1998) (X. Q. Yang, A. I. Mees, M. E. Fisher, and L. S. Jennings, eds.), Appl. Optim., vol. 39, Kluwer Academic, Dordrecht, 2000, pp. 167-179.

[18] G. M. Lee, D. S. Kim, and H. Kuk, Existence of solutions for vector optimization problems, Journal of Mathematical Analysis and Applications 220 (1998), no. 1, 90-98.

[19] G. M. Lee, D. S. Kim, B. S. Lee, and N. D. Yen, Vector variational inequality as a tool for studying vector optimization problems, Nonlinear Analysis 34 (1998), no. 5, 745-765.

[20] G. M. Lee and S. H. Kum, On implicit vector variational inequalities, Journal of Optimization Theory and Applications 104 (2000), no. 2, 409-425.

[21] S. Park, Some coincidence theorems on acyclic multifunctions and applications to KKM theory, Fixed Point Theory and Applications (Halifax, NS, 1991), World Scientific, New Jersey, 1992, pp. 248-277.

[22] T. D. Phuong, P. H. Sach, and N. D. Yen, Strict lower semicontinuity of the level sets and invexity of a locally Lipschitz function, Journal of Optimization Theory and Applications 87 (1995), no. 3, 579-594.

[23] T. W. Reiland, Nonsmooth invexity, Bulletin of the Australian Mathematical Society 42 (1990), no. 3, 437-446.

[24] R. T. Rockafellar, Convex Analysis, Princeton Mathematical Series, no. 28, Princeton University Press, New Jersey, 1970.

[25] X. Q. Yang, Generalized convex functions and vector variational inequalities, Journal of Optimization Theory and Applications 79 (1993), no. 3, 563-580.

Lucelina Batista Santos: Departamento de Matemática, Universidade Federal do Paraná, CP 19081 CEP, 81531-990 Curitiba, Paraná, Brazil

E-mail address: lucelina@mat.ufpr.br

Marko A. Rojas-Medar: Departamento de Matemática Aplicada, Universidade Estadual de Campinas, CP 6065, 13083-859 Campinas, São Paulo, Brazil

E-mail address: marko@ime.unicamp.br

Antonio Rufián-Lizana: Departamento de Estadística e Investigación Operativa, Universidad de Sevilla, 41012 Sevilla, Spain

E-mail address: rufian@us.es 


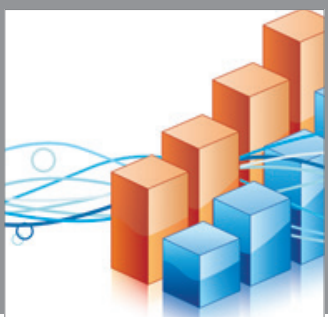

Advances in

Operations Research

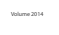

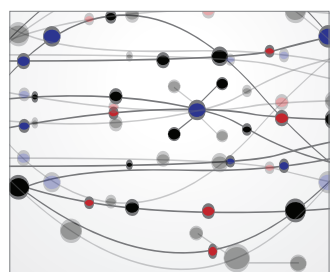

\section{The Scientific} World Journal
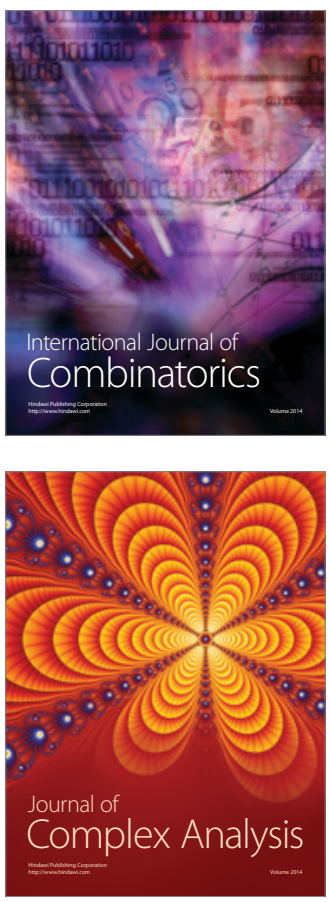

International Journal of

Mathematics and

Mathematical

Sciences
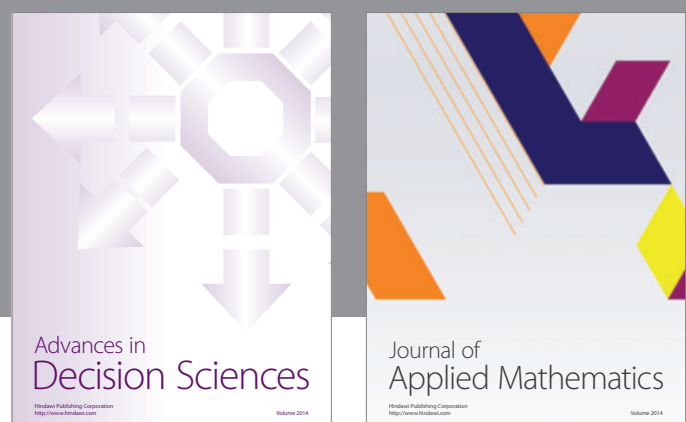

Journal of

Applied Mathematics
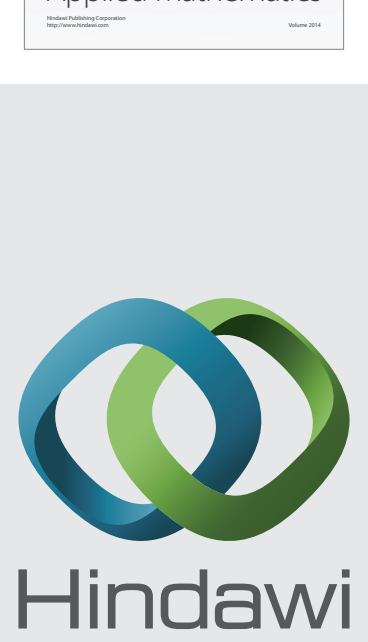

Submit your manuscripts at http://www.hindawi.com
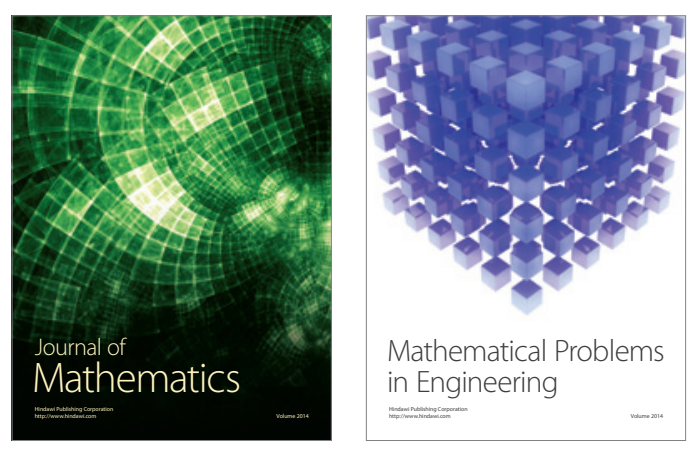

Mathematical Problems in Engineering
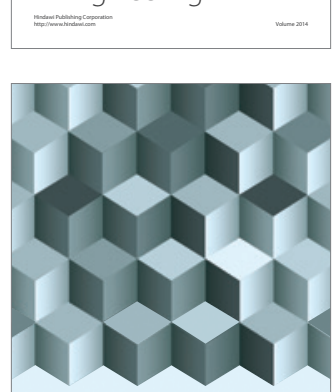

Journal of

Function Spaces
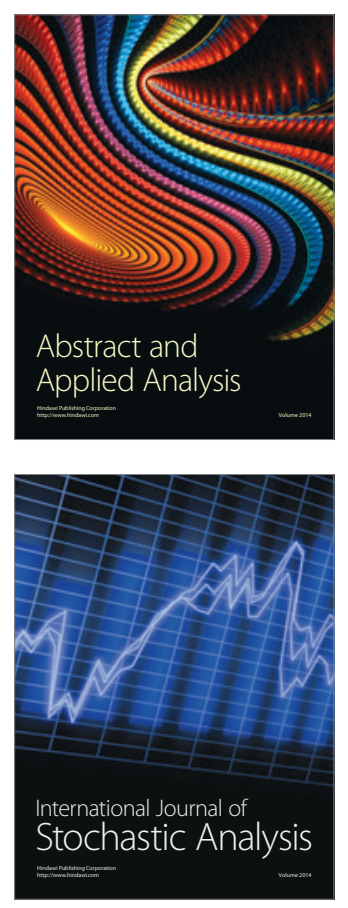

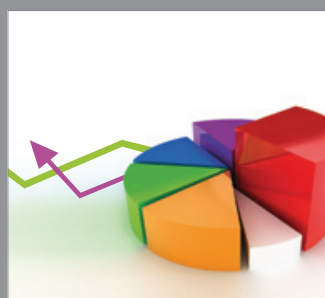

ournal of

Probability and Statistics

Promensencen
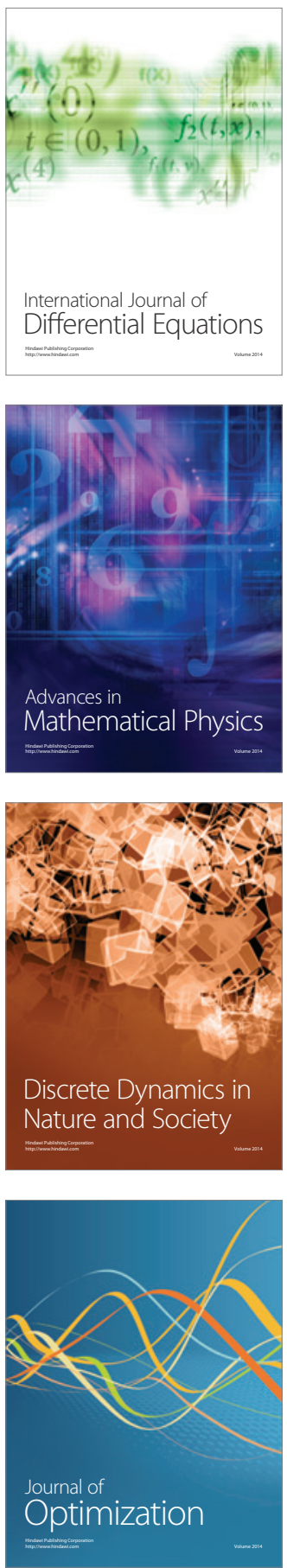